\title{
Initial Results from the Southern Edgeworth-Kuiper belt Survey
}

Rachel Moody and Brian Schmidt

Research School of Astronomy and Astrophysics, Australian National University, ACT 2602

Charles Alcock and Jeffrey Goldader

Department of Astronomy, University of Pennsylvania, PA, 19104

Tim Axelrod

Steward Observatory, University of Arizona

Kem H Cook and Stuart Marshall

Lawrence Livermore National Laboratory, Livermore, CA 94551-9900

\begin{abstract}
We present preliminary results of the analysis of the first 500 square degrees of the Southern Edgeworth-Kuiper belt Survey, a large sky survey which ran from January 2000 to January 2003 and comprises $2900 \square^{\circ}$ in total. Early tests predict that we should discover $\sim 10$ bright TNOs in our data, doubling the current known population.
\end{abstract}

Keywords: Kuiper belt - methods:observational - solar system:general - surveys

\section{Introduction}

The discovery of the Edgeworth-Kuiper belt (Jewitt \& Luu, 1993) opened up a new frontier in modern planetary astronomy. Currently more than 750 TNOs are recorded at the Minor Planet Center ${ }^{1}$ but the majority of surveys have had small sky coverage, making them relatively less sensitive to bright TNOs and so very few of these have been discovered.

However, surveying for bright, rare TNOs has the advantage that there will inevitably be pre-discovery images available which can help refine the orbital parameters. Another advantage is that all TNOs found in such a survey will most likely be good spectroscopic candidates.

Ivezic et al (2001) notes that obtaining a proper inventory of bodies in the outer Solar System requires a survey with large sky coverage, faint limiting magnitude, uniform and well defined detection limits in magnitude and proper motion, accurate multicolor photometry for taxonomy, and sufficient follow-up observations in order to obtain well determined orbits. In this work we report on the methodology and initial results from the Southern Edgeworth-Kuiper belt Survey (SEKS).

1 http:/www.harvard.com/MPC/TNOs.html

(c) 2003 Kluwer Academic Publishers. Printed in the Netherlands.

ConfProc.tex; 27/05/2003; 18:26; p.1 
This survey satisfies all but one of these requirements with the only shortcoming being a bright limiting magnitude.

\section{The Survey}

The Southern Edgeworth-Kuiper belt Survey (SEK) survey commenced January 1, 2000 and ran until January 18, 2003 when the telescope was destroyed by fire. The survey had several observational goals, including:

1. To uniformly survey the ecliptic in a $3^{\circ}$ wide band.

2. To observe fields up to $20^{\circ}$ away from the ecliptic in order to search for highly inclined TNOs.

3. To preferentially survey the southern latitudes which have not been well studied previously.

4. To make follow-up of TNO candidates a priority in order that the entire sample have well determined orbits.

5. To substantially increase the number of moderately large (diameter $>200 \mathrm{~km}$ ) objects known within the inner EKB.

The survey to date has satisfied the first three observational goals. Continuing analysis of the data will illustrate how well the survey satisfies the remaining goals.

Figure 1 is a plot of the entire region covered in our Survey. More than 5000 fields were observed covering $\sim 2900 \square^{\circ}$ on the sky. Each field was observed 3 times, the first two observations were taken $\sim 3$ hours apart while the 3rd observation was typically taken 24 hours later. The observations were taken with 5 minute exposures. This relatively short exposure time ensured that TNOs appeared as point sources while asteroids would appear as slightly extended images.

\subsection{Telescope System}

All observations were taken with the telescope used previously by the MACHO project (Alcock et al, 2000), which became fully automated in June 2001. It comprises a 50 inch aperture primary mirror, with a field of view of $0.5 \square^{\circ}$, and incorporates a dichroic element to allow

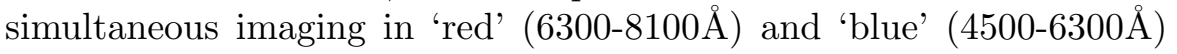
onto two CCD cameras (Hart et al., 1996). 


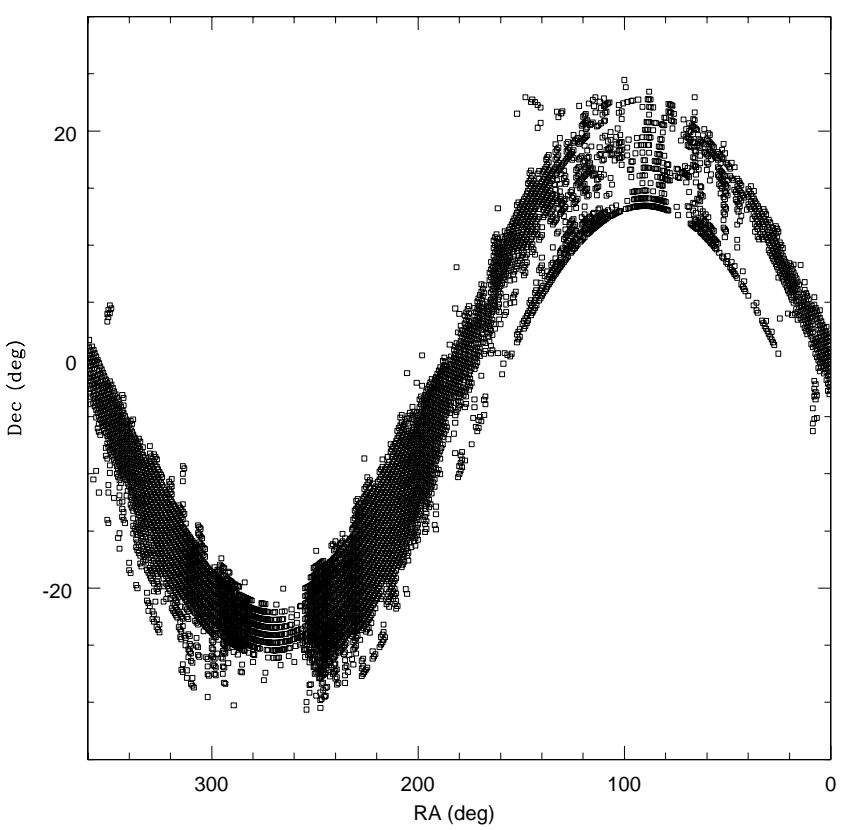

Figure 1. Plot of the fields observed by the Survey

\section{Data Analysis}

The observations are analyzed as two sets of pairs by the custom analysis pipeline, which was designed to simultaneously compare the red and blue images of the two observations. The steps involved in the pipeline included fitting a World Coordinate System, adding artificial objects, extracting all objects from each image and then comparing the objects from the 4 images to create a list of possible TNO candidates. These candidates are then checked by eye.

To examine the real-time detection efficiency of our survey, we created an artificial population of $10^{6}$ solar system bodies. These bodies have orbital elements uniformly distributed in parameter space in the ranges set out in Table I. Each body was also assumed to have an albedo of 0.04 in R-band and given a diameter, a colour offset, an amplitude of colour variation, and a period of 6 hours, representing rotation. The combination of these elements defines the position and brightness of an object at any given time. 
Table I.

\begin{tabular}{lc}
\hline Characteristics Defining Artificial Population & Range \\
\hline Semi-major Axis & $10-110 \mathrm{AU}$ \\
Inclination & $0-180^{\circ}$ \\
Eccentricity & $0-1$ \\
Argument of Perihelion & $0-360^{\circ}$ \\
Longitude of Ascending Node & $0-360^{\circ}$ \\
Mean Anomaly & $0-360^{\circ}$ \\
Diameter & $1-1200 \mathrm{~km}$ \\
B-V colour & $-0.1-1$ \\
Amplitude & $0,0.4,0.8$ \\
\hline
\end{tabular}

\subsection{TNO SElection CRiteria}

Typically, a candidate list contains $\sim 100$ objects, and this results in $\sim 1$ million possible combinations. We extract TNO candidates by finding objects which have 6 TNO-like characteristics over the course of the three observations. The selection criteria are:

1. Direction Since the motion of a TNO is due only to the Earth's motion, and the turn-around of TNOs is very short compared to the path of the orbit, all TNOs should follow an approximately straight line over the course of the observations. Thus, as a quick and efficient first step, we require that a TNO candidate must not reverse it's direction over the course of the observations.

2. Magnitude An object cannot vary greatly in magnitude in either the red or the blue image to be considered a TNO, so $\Delta$, the maximum difference in red and blue magnitudes, is calculated:

$$
\Delta \equiv \max _{i, j}\left\{m_{R_{i}}-m_{R_{j}}\right\}+\max _{i, j}\left\{m_{V_{i}}-m_{V_{j}}\right\}
$$

We require $\Delta<3$.

3. Difference in Angular Velocity As TNOs should have constant motion over the time of the observations, we require that the velocity of an object calculated between observations 1 and 2, and observations 2 and 3 , differ by less than $2 " / \mathrm{hr}$.

4. Maximum Angular Velocity A TNO in a circular orbit at 40 AU would have an angular velocity of $\sim 3 " / \mathrm{hr}$. In order to allow for objects on highly inclined and eccentric orbits, we allow objects to be TNO candidates if their angular motion is less than $7.5 \% / \mathrm{hr}$. 
5. Linear Motion Another consequence of a TNO's constant linear motion is that its path should be a straight line. We require that for an object to be considered a TNO, its path must not deviate from a straight line by more than 1".

6. Angle to the Ecliptic A TNO should have the same angle of motion to the ecliptic as does the vector defining the Earths motion. For the object to pass this criteria, it must have an angle within $20^{\circ}$ of the Earth's angle to the ecliptic (large enough to allow for TNOs with high inclinations).

The strength of these criteria is demonstrated in Table II, where the selection criteria were applied to field 4763 . When this field was processed 198, 48 and 91 candidates were found in each observation respectively, giving $\sim 850,000$ different combinations. Sequentially applying the 6 selection criteria reduced the number of TNO candidates to 19.

Table II. Table showing number of combinations remaining

\begin{tabular}{lc}
\hline Selection Criteria & Number of Combinations \\
\hline Data & 864,864 \\
Direction & 40,186 \\
Magnitude & 23,623 \\
Difference in Angular Velocity & 1,022 \\
Maximum Angular Velocity & 93 \\
Linear Motion & 81 \\
Angle to the Ecliptic & 19 \\
\hline
\end{tabular}

\section{Results}

Our initial analysis consisted of $\sim 500 \square^{\circ}$ on the sky. These fields were processed through the pipeline and all TNO candidates were checked by eye, but no TNOs were detected. In order to get a measure of the significance of this null result, we calculated the detection efficiency for all artificial objects added to the fields. This plot is shown in Figure 2. While this is only a very preliminary plot and much more analysis needs to take place but at this early stage the limiting magnitude for the survey is $m_{R} \sim 20$.

For each bin in Figure 2, we calculated the expected number of TNOs based on the cumulative luminosity function given by Trujillo et al. (2001), $\log \Sigma=0.66\left(m_{R}-23.32\right)$. By combining this equation with our detection efficiency and sky coverage we should have expected to find $2 \pm 1.6$ objects, so our null result is not significant. Another result 
of this calculation is that our survey expects to discover $\sim 10$ bright TNOs.

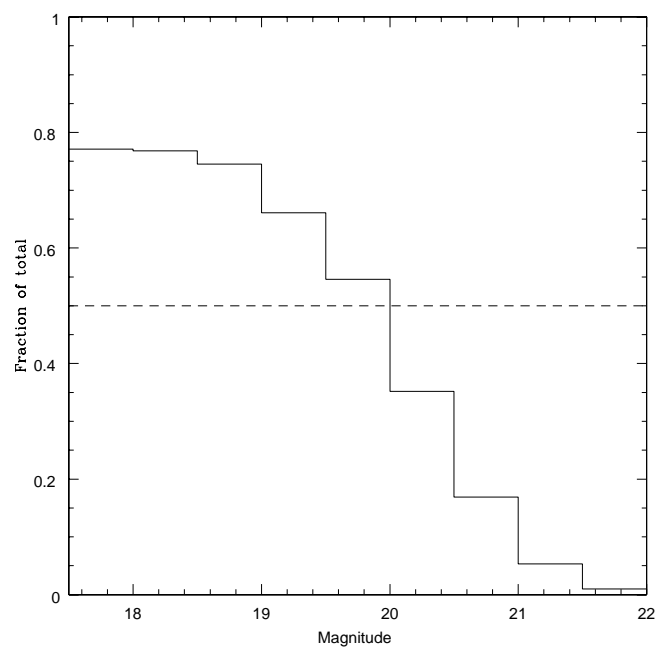

Figure 2. Detection Efficiency based on sample of 1000 fields. The dotted line indicates the $50 \%$ cutoff for the detection efficiency which for our sample is $m_{R} \sim 19.75$

\section{Conclusion}

All observations for our survey have now been completed, and analysis of the data is ongoing. Our initial analysis of the first $500 \square^{\circ}$ demonstrated that our current non-detection of a TNO is not significant, and that we should expect to detect $\sim 10$ objects in the entire dataset which would double the number of known bright TNOs.

\section{References}

Alcock, C. et al. The MACHO Project: Microlensing results from 5.7 years of Large Magellanic Cloud observations. apj, 542:281, 2000.

Hart, J. et al. The Telescope System of the MACHO Program. pasp, 220:108+, 1996.

Ivezić, Ž. et al. Solar System Objects Observed in the Sloan Digital Sky Survey Commissioning Data. aj, 122:2749-2784, 2001.

Jewitt, D. C. and Luu, J. X. Discovery of the Candidate Kuiper Belt Object 1992 $\mathrm{QB}_{1}$. nature, 362:, 1993.

Trujillo, C. A. and Jewitt, D. C. and Luu, J. X. Properties of the Trans-Neptunian Belt: Statistics from the Canada-France-Hawaii Telescope Survey. aj, 122:457473, 2001. 